am 13. - 17. September 2015 at Prague, Czech Republic

\title{
Can spatial audio support pilots? 3D-audio for future pilot- assistance systems
}

\author{
German Aerospace Center, Institute of Flight Guidance \\ Lilienthalplatz 7, 38108 Braunschweig
}

Christian Niermann, M. Sc.

phone: $+49(0) 531 / 295-2151$

Christian.Niermann@dlr.de

fax: $+49(0) 531 / 295-2550$

\begin{abstract}
:
With the introduction of new technologies in modern flight decks, the precision and efficiency of flight operations are increased. Most of the information provided to the cockpit crew is given via the human visual channel. Flying a helicopter or airplane is a combination of visually monitoring reference points to determine the actual position and movement of the aircraft and monitoring the information given visually to the pilots by the aircraft's systems.
\end{abstract}

Large visual-display units in state-of-the-art head-down glass cockpits provide a large amount of information. With helmet-mounted displays or head-up displays, used in civil and military aircrafts, the visual resources of the human are consumed intensely. In addition to the limited space in modern cockpits, also the cognitive ability of humans is limited, which creates new operational burdens and new kinds of failure modes in the overall human-machine system. Accordingly, research has to think about two topics: First, decreasing the visual workload to create free capacity in the human visual channel, which increases the information flow. Second, improving the performance of flight crews, e.g., during target localization, but simultaneously reducing workload and enhancing situation awareness in complex time-critical environments.

Today, only speech messages and simple warning sounds use the auditory channel. The sound applied in the cockpit does not convey any additional information using its location. Several studies have suggested sundry applications for the use of spatial audio in the cockpit. Audio research so far has been sparse in aviation and only covered spatial audio with a set of loudspeakers around the head of the pilot or simple left-right-volume difference in the headset of the pilot. We plan to use audio as a command system, to assist the flight crew to accomplish flight operations successfully, for example during takeoff and landing or in search and rescue operations. Being able to gain information about the location of targets without referring to head down displays will allow pilots to direct more of their visual-processing capacity to critical tasks that require them to look outside from the cockpit.

We programmed a software to play a 3-deminsional sound with a typical pilot's stereo headset and use this software to test how precisely a pilot can locate a sound object in a virtual space around him. Using a head tracker helps to determine whether the reference point of the proband moves with his head rotation in a cockpit environment or stays attached to the aircrafts heading. Two studies are planned. In the first step, 3D-audio is used as an addition to the visual channel to support the pilots in finding information faster and more accurately. A second study will determine whether 3D-audio can replace visual information.

As a first step toward our studies, this paper presents the current state of research concerning cockpit audio. Psychological and physiological limits of human beings are shown. Pros and cons of 3D-audio are compared and various possibilities how audio could support in the field of aviation are presented.

Christian Niermann joined the Institute of Flight Guidance of the German Aerospace Center (DLR) in 2014 as a researcher and PhD student. He studied "Human Factors" with focus on 
"Aviation and Space" at the University of Berlin where he graduated in 2013. He also holds a private pilot license. In his research, he focuses on new multi-modal human-machine interaction for future pilot assistance systems. 